\title{
In Vivo Relationship Between Hypoxia and Angiogenesis in Human Glioblastoma: A Multimodal Imaging Study
}

\author{
Keven Ferreira da Ponte ${ }^{* 1,2}$, David Hassanein Berro*1,2, Solène Collet ${ }^{1}$, Jean-Marc Constans ${ }^{1,3}$, Evelyne Emery ${ }^{2,4}$, \\ Samuel Valable ${ }^{1}$, and Jean-Sébastien Guillamo ${ }^{1,5,6}$ \\ ${ }^{I}$ Normandie University, UNICAEN, CEA, CNRS, ISTCT/CERVOxy Group, Caen, France; ${ }^{2}$ Department of Neurosurgery, University \\ Hospital of Caen, Caen, France; ${ }^{3}$ Department of Neuroradiology, University Hospital of Caen, Caen, France; ${ }^{4}$ Normandie University, \\ UNICAEN, INSERM, Inserm UMR-S U919, Caen, France; ${ }^{5}$ Department of Neurology, University Hospital of Caen, Caen, France; \\ and ${ }^{6}$ Department of Neurology, University Hospital of Nimes, Nimes, France
}

\begin{abstract}
Glioblastoma multiforme (GBM) is the most common and aggressive primary brain tumor. This aggressiveness is in part attributed to the closely interrelated phenomena tumor hypoxia and angiogenesis, although few in vivo data exist in human brain tumors. This work aimed to study hypoxia and angiogenesis, in vivo and in situ, in patients admitted with GBM using multimodal imaging. Methods: Twenty-three GBM patients were assessed by ${ }^{18} \mathrm{~F}$-fluoromisonidazole ( $\left.{ }^{18} \mathrm{~F}-\mathrm{FMISO}\right)$ PET and conventional and perfusion MRI before surgery. The level and location of hypoxia ( ${ }^{18} \mathrm{~F}-\mathrm{FMISO}$ uptake, evaluated by tumor-to-blood [T/B] ratio), vascularization (cerebral blood volume [CBV]), and vascular permeability (contrast enhancement after gadolinium injection) were analyzed. The spatial relationship between tumor hypoxia and angiogenesis was assessed by an overlap analysis of the volume of ${ }^{18} \mathrm{~F}$-FMISO uptake and the volumes of the high CBV regions and the contrast-enhancement regions. Results: A significant correlation was found between hypoxia and hypervascularization, especially for their maximum values (volume of maximal tumor hypoxia vs. relative CBV: $r=0.61, P=0.002$ ) and their volumes (hypoxia vs. hypervascularization: $r=0.91, P<$ $0.001)$. A large proportion of the high CBVs collocated with hypoxia (81.3\%) and with contrast enhancement (46.5\%). Conclusion: These results support the hypothesis of a tight association between hypoxia and angiogenesis. Our results suggest that there is insufficient tumor oxygenation in human GBM, despite increased tumor vascularization.
\end{abstract}

Key Words: glioblastoma; hypoxia; ${ }^{18} \mathrm{~F}-\mathrm{FMISO}$ PET; angiogenesis; MRI

J Nucl Med 2017; 58:1574-1579

DOI: 10.2967/jnumed.116.188557

G lioblastoma multiforme (GBM) is the most common primary brain tumor and one of the most aggressive forms of cancer in humans, with an estimated incidence of 2-3/100,000 in Europe and North America (1). Glioblastoma is particularly resistant to antitumor treatments, with a high rate of rapid recurrence and short survival (12-15 mo) $(2,3)$.

Received Jan. 12, 2017; revision accepted Mar. 22, 2017

For correspondence or reprints contact: Jean-Sébastien Guillamo, Service de Neurologie, CHU de Nimes, Place du Professeur Robert Debre, 30029 Nimes cedex 9, France.

E-mail: jeansebastien.GUILLAMO@chu-nimes.fr

${ }^{*}$ Contributed equally to this work.

Published online Jun. 8, 2017.

COPYRIGHT (C) 2017 by the Society of Nuclear Medicine and Molecular Imaging.
GBM is typically characterized by a central necrosis aspect surrounded by heterogeneous contrast enhancement (CE) after gadolinium injection on MRI $(4,5)$. These imaging features are supported by 2 pathophysiologic phenomena: tumor hypoxia and angiogenesis (6). Hypoxia induces the expression of several genes involved in angiogenesis, proliferation and survival, anaerobic metabolism, pH regulation, and migration of tumor cells (7). Angiogenesis is one of the most important consequences of the hypoxic signal, which leads to an increased vascularization that favors tumor nutrition and growth $(8,9)$. Furthermore, these tumors are heterogeneous, both between different patients and within the tumor itself (10). Therefore, an imaging approach is well suited for the characterization of the different tumor compartments.

PET is currently considered to be the most appropriate imaging method for assessing tumor hypoxia (8). This method sensitively and quantitatively estimates local concentrations of radioactive molecules, such as nitroimidazole derivatives labeled with ${ }^{18} \mathrm{~F}$, used as markers of hypoxia. Among the nitroimidazole derivatives already synthesized, ${ }^{18} \mathrm{~F}$ fluoromisonidazole ( ${ }^{18} \mathrm{~F}$-FMISO) is the most used and studied radiotracer in human brain tumors. These compounds exclusively accumulate in hypoxic viable cells after reduction reactions in the absence of oxygen, necrotic cells lacking the necessary enzymatic activity $(8,11,12)$. This uptake is detected when pO2 is less than $10 \mathrm{~mm} \mathrm{Hg}$ (13).

MRI plays an increasingly important role in the in vivo characterization of tumor vascularization, representative of the extent of angiogenesis. Perfusion MRI (through the use of the first pass of a contrast agent, also called dynamic susceptibility contrast MRI) assesses changes in tumor microvascularization. The intensity of the signal is dependent on the concentration of gadolinium (Gd) in the vessels, and the number and diameter of the vessels (14). Image postprocessing can determine several parameters including the relative cerebral blood volume (rCBV) in each slice and for each voxel.

Experimental data have shown a strong link between hypoxia and angiogenesis $(7,15,16)$. Although it is widely acknowledged that tumor angiogenesis is triggered by hypoxia, few data exist of this association in vivo in human GBM. A recent multicenter study found that pretreatment abnormal tumor vasculature (as measured by MRI) and hypoxia (as measured by ${ }^{18} \mathrm{~F}$-FMISO PET) were associated with worse survival in patients with newly diagnosed GBM (17). Specifically, they found that increased tumor perfusion, vascular volume, vascular permeability, and hypoxia are negative prognostic markers, all of which can be measured safely and reliably using MRI and ${ }^{18}$ F-FMISO PET (17).

This work sought to study, in vivo and in situ, the spatial relationship between hypoxia and angiogenesis in GBM using ${ }^{18} \mathrm{~F}-\mathrm{FMISO}$ PET 
imaging and advanced MRI techniques. The purpose of the work was to characterize the relationships between hypoxia and angiogenesis markers with quantitative multiparametric and qualitative spatial analysis.

\section{MATERIALS AND METHODS}

\section{Patient Population}

Patients were included between October 2010 and May 2012, in the University Hospital of Caen, Caen, France. The study was approved by the ethics committee CPP (Comité de Protection des Personnes) Nord-Ouest III and AFSSAPS (Agence française de sécurité sanitaire des produits de santé) and registered under EUDRACT no. 2009-015543-16 and ClinicalTrials. gov NCT01200134. All the patients signed an informed consent form.

Patients with a presumed diagnosis of glioblastoma underwent an MRI in the University Hospital of Caen and a ${ }^{18} \mathrm{~F}$-FMISO PET at the Cyceron Biomedical imaging platform (Caen, France) within the same week. The patients then underwent surgical resection or biopsy. The diagnosis of glioblastoma was established by an experienced neuropathologist of the University Hospital of Caen, according to the World Health Organization 2007 criteria.

\section{F-FMISO PET Examination}

${ }^{18} \mathrm{~F}$-FMISO radiotracer was produced by the LDM-TEP group (UMR 6301 ISTCT) as previously described (18). Images were acquired using a GE Healthcare Discovery RX VCT HD scanner (CYCERON Biomedical Imaging Platform, spatial resolution of $6 \mathrm{~mm}$ in full width at half maximum $), 2 \mathrm{~h}(120.95 \pm 2.77 \mathrm{~min})$ after radiotracer injection at a dose of $5 \mathrm{MBq} / \mathrm{kg}$. Blood samples were taken immediately before and after image acquisition (duration of scan, $20 \mathrm{~min}$ ), and the mean value was used for tumor-to-blood (T/B) ratio. The attenuation-corrected images were reconstructed with an ordered-subset expectation maximization 2-dimensional algorithm ( 9 subsets and 2 iterations) and filtered in 3 dimensions with a Butterworth filter. For image normalization and quantification, we used the T/B method ( $\mathrm{T} / \mathrm{B}=$ radiotracer fixation in tumor $[\mathrm{kBq} / \mathrm{mL}] /$ measured mean blood activity $[\mathrm{kBq} / \mathrm{mL}]$ ) as described elsewhere (19).

\section{MRI Examination}

MRI was performed on a GE Healthcare Signa HDxt 1.5T machine. The following MRI sequences were used: standard anatomic sequences, including axial T1-weighted and axial fluid-attenuated inversion recovery (FLAIR) sequences (24 slices; slice spacing, 5.5; pixel resolution, $0.47 \times 0.47 \mathrm{~mm}$; repetition time [TR]/echo time [TE], 450/11 ms for T1-weighted and 9,602/150 ms for FLAIR); a dynamic susceptibility contrast first-pass perfusion sequence; and a 3-dimensional T1-weighted sequence (124 slices; slice spacing, 1.5; pixel resolution, $1.01 \times 1.01 \mathrm{~mm}$; TR/TE, $14 / 3 \mathrm{~ms}$ ).

For the perfusion sequence, dynamic $\mathrm{T} 2 *$ gradient-echo weighted images were acquired immediately before, during, and after the first pass of the gadolinium intravenous contrast, injected at a dose of 0.1 $\mathrm{mmol} / \mathrm{kg}$ (14 slices, 35 repetitions; slice spacing, $7 \mathrm{~mm}$; pixel resolution, $2.19 \times 2.19 \mathrm{~mm}$; TR/TE, 2,280/60 ms). Then a 3-dimensional T1-weighted sequence was used to evaluate gadolinium enhancement (T1Gd sequence) as a reflection of vessel permeability. The cerebral blood volume (CBV) maps were computed with ImageJ software (National Institutes of Health, 2013), according to the method published by Collet et al. (20).

\section{Image Processing and Parameter Calculation}

MRI sequences and CBV maps were coregistered with ${ }^{18} \mathrm{~F}-\mathrm{FMISO}$ PET images with trilinear interpolation, rigid matching, and normalized mutual information using PMOD software (version 3.1; PMOD Technologies Ltd., 2010).

Three regions of interest (ROIs) were manually delimited at the tumor site on MRI: radiologic necrosis region, corresponding to the central hypointensity on T1Gd sequences, considered a zone of macroscopic necrosis; classic tumor region, representing the tumor areas limited by the signal hyperintensity on T1Gd sequences, including the radiologic necrosis region; and total tumor region, corresponding to all the areas of hyperintensity on FLAIR sequences, including the classic tumor region (Fig. 1). Thereafter, 2 other ROIs were calculated from the previous 3: exclusive FLAIR region, consisting of the FLAIR hyperintensity beyond the T1Gd hyperintensity (total tumor region - classic tumor region), considered a region of brain edema and invasion; and CE region (classic tumor region - radiologic necrosis region). The latter is the region of gadolinium leakage due to the abnormal vascular permeability $(12,14,21)$. An ROI was defined in contralateral healthy cerebral parenchyma (in the normal-appearing white matter) for creation of normalized $\mathrm{CBV}$ values (rCBV) and volumes segmentation. These different ROIs were transposed onto all modalities (Fig. 1).

Next, the following parameters were calculated for each tumor and each ROI: first, mean (rCBVmean) and maximal (rCBVmax) relative $\mathrm{CBV}$, defined as the ratio between mean or maximal CBV of a tumor ROI and the mean $\mathrm{CBV}$ of the contralateral ROI (22); and second, $\mathrm{T} / \mathrm{Bmean}$, and $\mathrm{T} / \mathrm{Bmax}$, defined as mean and maximal values of $\mathrm{T} / \mathrm{B}$ of a tumor ROI.

To assess the spatial relationships between the ROIs, a comparison was made between the volume of ${ }^{18} \mathrm{~F}$-FMISO uptake and tumor volumes corresponding to the areas of high CBV and CE. The following volumes were established using semiautomatic segmentation. Hypoxic tumor volume is the volume obtained after thresholding the ${ }^{18} \mathrm{~F}-\mathrm{FMISO}$ images by the contralateral 95 centile. Thus, the tumor pixels were
FIGURE 1. Delimitation of different ROIs. T1Gd images (A) were used to calculate the following ROls: classic tumor region ( $\mathrm{B}$, cyan), radiologic necrosis region ( $\mathrm{T} 1 \mathrm{Gd}$ central hyposignal; $\mathrm{B}$ magenta), and, by subtraction, CE region (C). FLAIR images (D) were used to calculate the total tumor region ( $D$, yellow) and exclusive FLAIR region ( $D$, region between yellow and cyan). ROIs have been transposed on modalities $(E$ and $F)$ for calculating parameters. Then, a semiautomatic segmentation was performed for high-CBV $(G)$ and $-{ }^{18} \mathrm{~F}-\mathrm{FMISO}$ uptake $(\mathrm{H})$ volumes. 
considered hypoxic when their value was higher than the values of $95 \%$ of pixels of the normal brain tissue (contralateral ROI) (Fig. 1). Hypervascularized tumor volume is the volume resulting from thresholding CBV maps by the contralateral 95 centile, with suppression of pixels corresponding to normal cerebral vessels, anatomically identified on the T1Gd sequences in brain fissures (Fig. 1). The volume of maximal tumor hypoxia results from thresholding the ${ }^{18} \mathrm{~F}-\mathrm{FMISO}$ images by the tumor 95 centile (total tumor regions), that is, corresponding to $5 \%$ of the most intense pixels (most hypoxic) within the tumor. The volume of maximal tumor vascularization is the volume corresponding to $5 \%$ of the most intense pixels on the CBV maps (most vascularized) within the tumor. And last, the permeable tumor volume is the volume of the zone of abnormal vascular permeability, calculated from the $\mathrm{CE}$ regions.

\section{Statistical Analysis}

Data are expressed as mean \pm SD. Comparisons between quantiand associations sought with Pearson correlation tests. Comparisons of categoric variables were performed using ANOVA and the Student $t$ test for paired samples. The statistical significance threshold was a $P$ value of 0.05 . All statistical analyses were performed using JMP software (SAS Institute Inc., 2012). The spatial relations were evaluated with an object-based colocalization analysis with measure of overlap rates between the volumes. To present this graphically, a Venn diagram was performed using a JMP add-in.

\section{RESULTS}

\section{Patients}

Twenty-three patients were included (5 women and $18 \mathrm{men}$ ), with a mean age of $61.4 \mathrm{y}$ (range, 28-79 y). All the patients had a tative variables were performed using simple linear regression tests

confirmed diagnosis of glioblastoma (World Health Organization grade IV) after surgical biopsy or resection.

\section{Hypoxic Profile of Tumors with ${ }^{18}$ F-FMISO PET Imaging}

All GBM patients presented ${ }^{18} \mathrm{~F}$-FMISO uptake, with a $\mathrm{T} / \mathrm{Bmean}$ in total tumor regions of $1.17 \pm 0.22$ (vs. $1.04 \pm 0.16$ in the contralateral normal brain tissue, $P<0.001$ ). Hypoxic tumor volumes presented wide variations between patients, with a mean of $52.06 \pm 38.23 \mathrm{~cm}^{3}$. Across all the tumors, $45 \%$ of the hypoxic volume was localized in the $\mathrm{CE}$ region, $40 \%$ in the exclusive FLAIR region, and $15 \%$ in the radiologic necrosis region. Maximal tumor hypoxia showed a significantly different distribution, with $61 \%$ of this volume localized in the CE region and only $8 \%$ within the exclusive FLAIR region, but with $31 \%$ in the radiologic necrosis region. Figure 2 shows an illustrative case of hypoxia localization.

\section{Vascularization Profile of Tumors with MRI}

Two patients could not be included in CBV analysis (no perfusion MRI available). For the other 21 patients, CBV was significantly higher in the tumor than the contralateral side $(0.13 \pm$ 0.05 vs. $0.10 \pm 0.03, P<0.001)$, resulting in an rCBVmean in total tumor regions of $1.41 \pm 0.31$. Hypervascularized tumor volume was on average $26.35 \pm 18.9 \mathrm{~cm}^{3}$. The permeable tumor volume was $30.15 \pm 26.25 \mathrm{~cm}^{3}$. Concerning the localization of hypervascularization zones within the tumor, $46 \%$ was within the CE region, $39 \%$ in the exclusive FLAIR region, and $15 \%$ in the radiologic necrosis region. Maximal tumor vascularization showed a similar distribution, with $54 \%$ within the $\mathrm{CE}$ region, $32 \%$ within the exclusive FLAIR region, and $14 \%$ in the radiologic necrosis region. Figure 2 shows an illustrative case of the localization of hypervascularization zones.

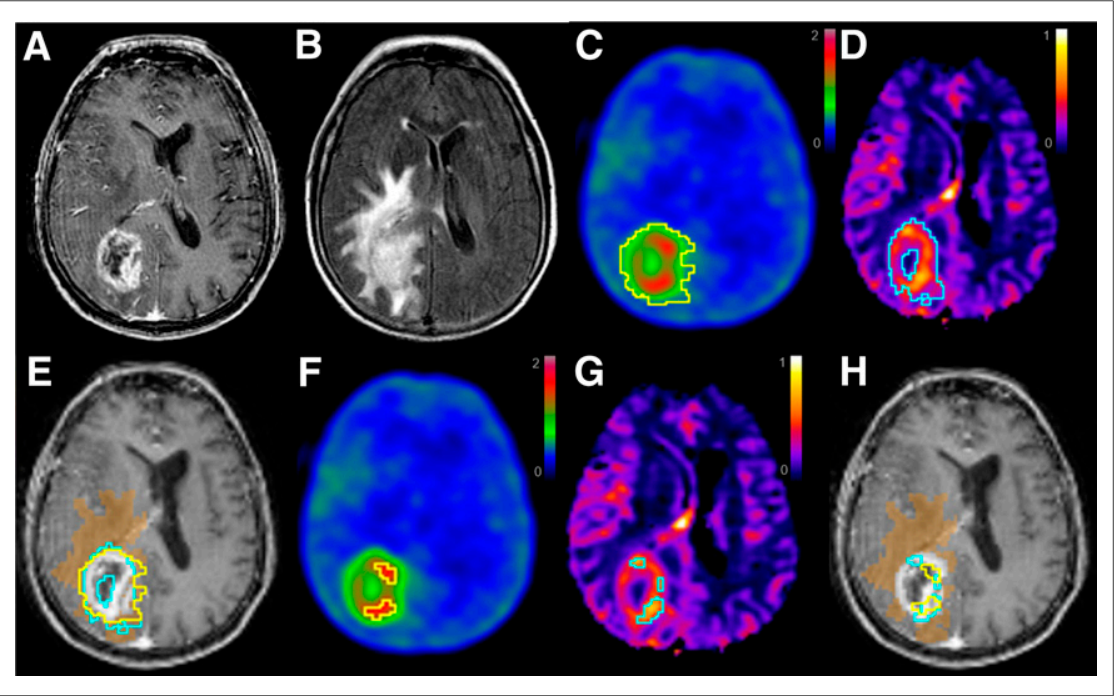

FIGURE 2. Right occipital glioblastoma. (A) MRI T1Gd sequence, illustrating typical appearance of glioblastoma with peripheral hypersignal reflecting extravasation of gadolinium, associated with central hyposignal (radiologic necrosis). (B) FLAIR sequence showing hyperintensity that far exceeds volume of CE. (C) ${ }^{18} \mathrm{~F}-\mathrm{FMISO}$ PET image (T/B) revealing significant uptake within tumor. Yellow outline shows automatic thresholding by contralateral 95 centile, which yielded hypoxic tumor volume. (D) CBV map also shows widespread tumor vascularization. Cyan outline represents threshold by contralateral 95 centile, which has produced hypervascularized tumor volume. $(E)$ Representation of different volumes on T1Gd sequence after coregistration of all modalities. White area represents $C E$ compartment, and brown zone represents exclusive FLAIR region. (F-H) Automatic thresholding on same images of $C, D$, and $E$ of volume of maximal tumor hypoxia $(F)$ and volume of maximal tumor vascularization $(G)$ by tumoral 95 centile (5\% of most intense pixels).

\section{Relationship Between Hypoxia and Angiogenesis}

Quantitative Analysis. Given the finding that a considerable fraction of ${ }^{18} \mathrm{~F}$-FMISO uptake and high CBV was located outside the $\mathrm{CE}$ region, an analysis was performed on the relationships between the degree of hypoxia and vascularization in total tumor regions. The classic tumor volume (CE and radiologic necrosis regions together) was evaluated separately. There was a positive correlation between the maximum values of hypoxia and vascularization within the entire tumor (T/Bmax vs. rCBVmax: $r=$ $0.61, P=0.002)$. The correlation was similar when the classic tumor region was assessed ( $r=0.77, P<0.001$; Fig. 3 A). There was a positive correlation between $\mathrm{T} / \mathrm{Bmean}$ and rCBVmean ( $r=0.57, P=0.007)$ within the hypervascularized tumor volume.

Finally, there was a strong positive correlation between hypoxic tumor volumes and hypervascularized tumor volumes ( $r=$ 0.91, $P<0.001$; Fig. 3B) and permeable tumor volumes $(r=0.93, P<0.001)$. All correlations are shown in Table 1.

Spatial Analysis. To understand the spatial relationships between hypoxia and angiogenesis, volume overlap analysis was performed, 
FIGURE 3. Scatterplot with linear regression lines illustrating relationship between uptake of ${ }^{18} \mathrm{~F}-\mathrm{FMISO}$ and vascularization. (A) Correlation between maximum values of ${ }^{18} \mathrm{~F}-\mathrm{FMISO}$ uptake expressed as T/B and rCBV in glioblastomas (classic tumor volume). (B) Relationship between hypoxic tumor volume $\left({ }^{18} \mathrm{~F}-\mathrm{FMISO}\right.$ uptake thresholded by contralateral 95 centile) and hypervascularized tumor volume (high CBV thresholded by contralateral 95 centile).

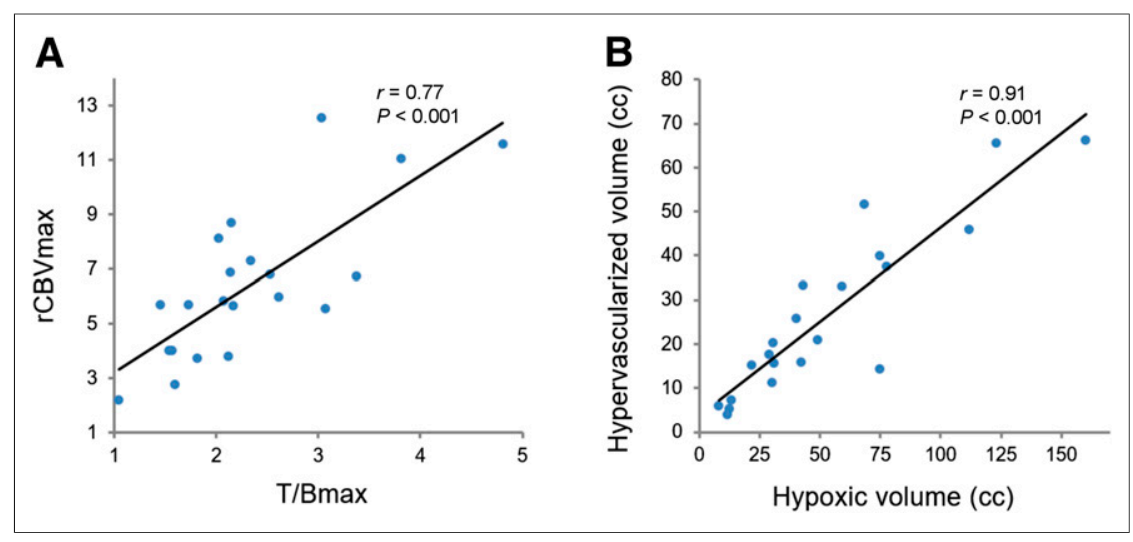

\section{DISCUSSION}

Our study is the first, to our knowledge, to analyze the spatial relationship between ${ }^{18} \mathrm{~F}$ FMISO PET and CBV maps in GBM, before surgery. Moreover, we examine vascularization and hypoxia within the different tumor compartments (i.e., infiltration area, CE, radiologic necrosis) and demonstrate the tight interrelations between hypoxia and angiogenesis in GBM. The analysis of the association between imaging parameters demonstrated a strong positive correlation between tumor vascularization and hypoxia, both in terms of volumes and intensity, particularly for maximum values. This is intriguing when considering that the most vascularized tumors should have the higher oxygen supply and therefore less hypoxia. However, blood vessels in glioblastomas are structurally and functionally abnormal, responsible for an ineffective per-

taking into account the size and location of volumes of hypoxia, hypervascularization, and permeability, within each tumor. When hypoxic and hypervascularized tumor volumes were compared, there was a large colocalization, with a mean overlap of ${ }^{18} \mathrm{~F}$-FMISO uptake zone by zone of high CBV of $49 \%$. In other terms, nearly $50 \%$ of hypoxic tumor volumes were covered by hypervascularized tumor volumes. Inversely, more than $80 \%$ of hypervascularized volumes were covered by hypoxic volumes. Overlap zones were seen to largely encompass the areas of maximal hypoxia, because on average $68 \%$ of volumes of maximal tumor hypoxia were covered by hypervascularized volumes. Conversely, only $18 \%$ of maximal tumor hypoxia coincides with the volume of maximal vascularization. Table 2 shows the overlap rates between the volumes of tumor hypoxia and hypervascularization, as well as these volumes compared with the permeability volume. Figure 4 illustrates these results using a Venn diagram, which shows the relationship and proportion between the sets of tumor volumes (the area of the circles is proportional to the mean value of the tumor volumes, and the common areas are proportional to the overlap rates between volumes). fusion and leading to tumor hypoxia $(9,14,23,24)$.

Indeed, the study conducted by Brühlmeier et al. (25) on 11 patients showed ${ }^{18} \mathrm{~F}$-FMISO uptake in both hypo- and hyperperfused tumor regions, suggesting that hypoxia may develop independently of tumor perfusion, estimated by ${ }^{15} \mathrm{O}-\mathrm{H}_{2} \mathrm{O}$ PET.

A second hypothesis could be that the hypoxic zones might not be located in the same areas as hypervascularization zones, given the intratumoral heterogeneity of glioblastoma. To test this hypothesis, a spatial analysis was conducted on the localization of ${ }^{18} \mathrm{~F}-\mathrm{FMISO}$ uptake and CBV maps. Our results showed an important overlap between hypervascularization and hypoxic zones.

When the different tumor compartments are analyzed more precisely, some interesting results were also observed. Hypoxia, as assessed by its maximum values (T/Bmax, volume of maximal tumor hypoxia), tends to be greater toward the center of the tumor. A higher proportion of intense hypoxia was observed in the $\mathrm{CE}$ region and even in the central radiologic necrosis region, with only a small proportion found beyond the classic tumor region. Conversely, the CBV tends to be higher in the periphery, with a substantial proportion of high $\mathrm{CBV}$

TABLE 1

Correlation Between Different Imaging Parameters ( ${ }^{18} \mathrm{~F}-\mathrm{FMISO}$ PET and MRI)*

\begin{tabular}{|c|c|c|c|c|}
\hline \multirow[b]{2}{*}{ Parameter } & \multicolumn{2}{|c|}{ Total tumor regions } & \multicolumn{2}{|c|}{ Classic tumor regions } \\
\hline & $r$ & $P$ & $r$ & $P$ \\
\hline \multicolumn{5}{|l|}{ Maximal and mean value } \\
\hline T/Bmax vs. rCBVmax & 0.61 & $0.002^{\dagger}$ & 0.77 & $<0.001^{\dagger}$ \\
\hline T/Bmean vs. rCBVmean & 0.29 & 0.185 & 0.38 & 0.079 \\
\hline \multicolumn{5}{|l|}{ Volume } \\
\hline Hypoxic tumor volume vs. hypervascularized tumor volume & & & 0.91 & $<0.001^{\dagger}$ \\
\hline Hypoxic tumor volume vs. permeable tumor volume & & & 0.93 & $<0.001^{\dagger}$ \\
\hline Hypervascularized tumor volume vs. permeable tumor volume & & & 0.88 & $<0.001^{\dagger}$ \\
\hline \multicolumn{5}{|c|}{$\begin{array}{l}{ }^{*} \text { Pearson correlation coefficients }(r) \text { with corresponding } P \text { values. } \\
{ }^{\dagger} P \text { values are statistically significant. } \\
\text { Hypoxic tumor volume }=18 \mathrm{~F}-\mathrm{FMISO} \text { uptake volume thresholded by contralateral } 95 \text { centile; hypervascularized tumor volume = high } \\
\text { BV thresholded by contralateral } 95 \text { centile; permeable tumor volume = volume of CE region. }\end{array}$} \\
\hline
\end{tabular}


TABLE 2

Mean Overlap Rate Between Hypoxic, Hypervascularized, and Permeable Tumor Volumes

\begin{tabular}{lll}
\hline \multicolumn{1}{c}{ Recovery of } & \multicolumn{1}{c}{ By } & \multicolumn{1}{c}{ Mean \% } \\
\hline Hypoxic tumor volume & Hypervascularized tumor volume & 48.8 \\
\hline Volume of maximal tumor hypoxia & Permeable tumor volume & 44.3 \\
& Hypervascularized tumor volume & 68.0 \\
\hline Hypervascularized tumor volume & Permeable tumor volume & 60.8 \\
\hline & Volume of maximal tumor vascularization & 18.0 \\
\hline & Hypoxic tumor volume & 81.3 \\
\hline
\end{tabular}

Hypoxic tumor volume $=18 \mathrm{~F}-\mathrm{FMISO}$ uptake volume thresholded by contralateral 95 centile; hypervascularized tumor volume $=$ high CBV thresholded by contralateral 95 centile; permeable tumor volume = volume of $\mathrm{CE}$ region; volume of maximal tumor hypoxia $={ }^{18} \mathrm{~F}-$ FMISO uptake volume thresholded by tumoral 95 centile (most intense $5 \%$ pixels); volume of maximal tumor vascularization $=$ high $\mathrm{CBV}$ thresholded by tumoral 95 centile.

values (30\% of the volume of maximal tumor vascularization) verified beyond the classic tumor region. This suggests that in the exclusive FLAIR region, angiogenesis is marked by effective tumor vascularity, with less permeability (no CE) and more oxygenation (less ${ }^{18} \mathrm{~F}$ FMISO uptake), possibly evolving secondarily into a typical abnormal vascularization with tumor growth. Hypoxia is known to induce the expression of angiogenic factors, such as vascular endothelial growth factor (VEGF), leading to vascular proliferation to combat the low oxygen availability (8). Our data indicate that while in some regions vascularization is effective for oxygen supply (zones without overlap between hypervascularization and hypoxia), in most

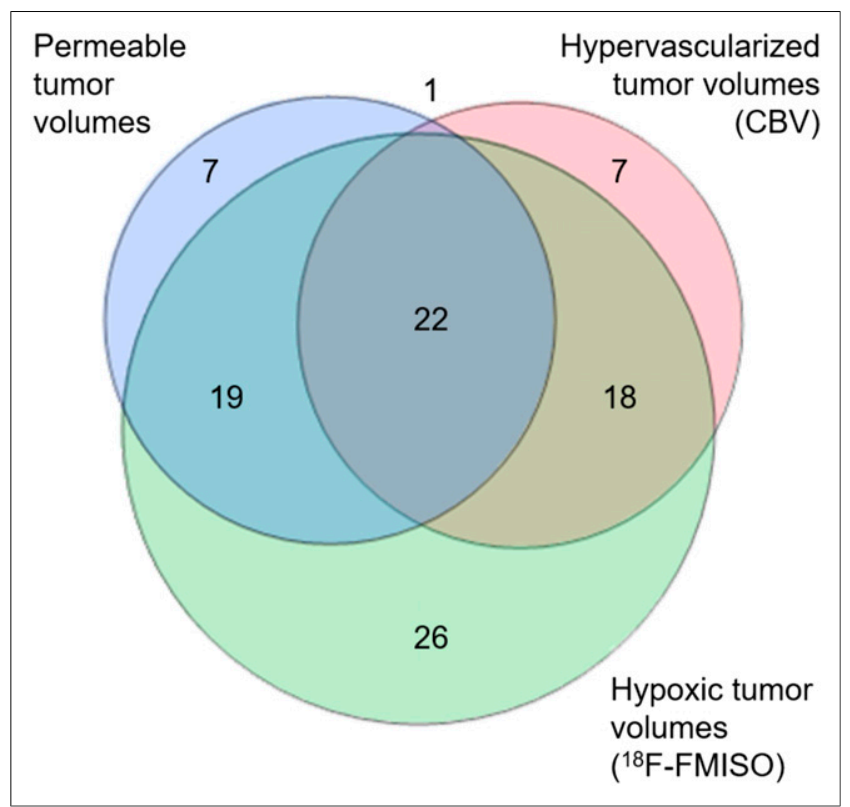

FIGURE 4. Venn diagram representing relationship between hypoxic $\left({ }^{18} \mathrm{~F}-\mathrm{FMISO}\right.$ uptake), hypervascularized (high CBV), and permeable (CE) tumor volumes of study population. Numbers and colored areas indicate proportions between different volumes and overlap rates (Table 2). There is greater volume of ${ }^{18} \mathrm{~F}$-FMISO uptake, with large overlap with both CBV and permeable tumor volume. There is also overlap between hypervascularized and permeable tumor volumes, corresponding to almost $50 \%$ of these volumes. of the tumor, angiogenesis is abnormal and insufficient to reverse hypoxia. Histopathologic analysis of GBM often shows immature nonfunctional blood vessels, with regions of arteriovenous shunts and thrombosis $(9,14,23,24)$.

Some practical inferences can be derived from the compartments analysis. First, tumor hypoxia and hypervascularization were more frequent and intense in the $\mathrm{CE}$ region (abnormal vascular permeability zone), which strengthens the link between these 2 phenomena. Second, it also confirms that the CE region, which is most frequently the treatment target zone of glioblastomas whether by surgery or radiotherapy, does not represent the only active area of the tumor. Indeed, hypoxia and angiogenesis occur beyond this region, along with well-documented brain tumor invasion. Finally, the presence of ${ }^{18}$ F-FMISO uptake and hypervascularized zones within the central area of necrosis illustrates the lack of specificity of the radiologic necrosis definition. This area, often heterogeneous, also comprises viable and active cells. Therefore, multimodal imaging could provide a better characterization of the classic macroscopic radiologic necrosis.

In the literature, we found only 4 studies that assessed the relationship between hypoxia and angiogenesis in glioblastomas using in vivo noninvasive imaging techniques. Swanson et al. (12) assessed the relationship between the uptake of ${ }^{18} \mathrm{~F}$-FMISO and $\mathrm{CE}$ area (T1G) in 11 patients with GBM. They found a significant correlation only when the surface areas of the hypoxic volume and the CE volume were compared.

In another study, Cher et al. (26) studied the link between the uptake of ${ }^{18}$ F-FMISO with the uptake of ${ }^{18}$ F-FDG and molecular markers of hypoxia and angiogenesis in 15 patients with glioma (7 GBM). These authors were able to demonstrate a significant correlation between hypoxia in PET imaging and the expression of VEGF receptor 1, but not with that of VEGF. However, later Kawai et al. (27) demonstrated a significant but weak correlation between the expression of VEGF and the ${ }^{18} \mathrm{~F}$-FMISO uptake in newly diagnosed high-grade glioma, including 18 glioblastomas.

Recently, Gerstner et al. (17) conducted a multicenter study to determine the prognostic value of the ${ }^{18} \mathrm{~F}$-FMISO PET and perfusion MRI parameters in patients with newly diagnosed GBM. In a secondary analysis, they explored the correlation between vascular parameters in MRI and the ${ }^{18} \mathrm{~F}-\mathrm{FMISO}$ PET uptake in $42 \mathrm{GBM}$ patients. There was a positive correlation between elevated $\mathrm{CBF}$ and 
the hypoxic volume. However, some limitations of this study prevent further analysis. Imaging studies were performed after surgery, thus the numerous postoperative artifacts and smaller tumor volumes, more sensitive to partial-volume effects, may hinder image evaluation. In addition, different PET scanners and MRI machines used in different centers and the dynamic susceptibility contrast MRI analysis limited to the enhancing tumor regions precludes an accurate comparison between the parameters of hypoxia and angiogenesis.

Our study is the only one to date assessing the spatial relationship in vivo between hypoxia and angiogenesis in GBM patients using ${ }^{18} \mathrm{~F}$-FMISO PET and perfusion MRI performed preoperatively. Nevertheless, it has some limitations. The number of patients is still limited, although this is one of the largest series of glioblastomas analyzed by multimodal imaging so far described. Furthermore, it should be noted that our study focused on hypoxia and angiogenesis with their spatial relationship at a given time, but their temporal relationship cannot be determined.

\section{CONCLUSION}

This study confirms, in vivo, through noninvasive multimodal imaging, the hypoxic and angiogenic hallmark of human glioblastomas and indicates interdependence between these 2 phenomena. Tumors with higher uptake of ${ }^{18} \mathrm{~F}$-FMISO are generally tumors with higher $\mathrm{CBV}$ and volume of $\mathrm{CE}$.

In contrast, tumor hypervascularization as assessed by the CBV on MRI in glioblastomas is not necessarily a source of effective tissue oxygenation and appears instead to be the result of abnormal tumor angiogenesis.

\section{DISCLOSURE}

This study was funded by the "Institut National Du Cancer" (INCA). No other potential conflict of interest relevant to this article was reported.

\section{ACKNOWLEDGMENT}

We thank Sarah Kabani for editing the manuscript.

\section{REFERENCES}

1. Bartek J, Ng K, Bartek J, Fischer W, Carter B, Chen CC. Key concepts in glioblastoma therapy. J Neurol Neurosurg Psychiatry. 2012;83:753-760.

2. Lacroix M, Abi-Said D, Fourney DR, et al. A multivariate analysis of 416 patients with glioblastoma multiforme: prognosis, extent of resection, and survival. J Neurosurg. 2001;95:190-198.

3. Tait MJ, Petrik V, Loosemore A, Bell BA, Papadopoulos MC. Survival of patients with glioblastoma multiforme has not improved between 1993 and 2004: analysis of 625 cases. Br J Neurosurg. 2007;21:496-500.

4. Asari S, Makabe T, Katayama S, Itoh T, Tsuchida S, Ohmoto T. Assessment of the pathological grade of astrocytic gliomas using an MRI score. Neuroradiology. 1994;36:308-310.

5. Ideguchi M, Kajiwara K, Goto $\mathrm{H}$, et al. MRI findings and pathological features in early-stage glioblastoma. J Neurooncol. 2015;123:289-297.
6. Barajas RF, Phillips JJ, Parvataneni R, et al. Regional variation in histopathologic features of tumor specimens from treatment-naive glioblastoma correlates with anatomic and physiologic MR imaging. Neuro-oncol. 2012;14:942-954.

7. Sharp FR, Bernaudin M. HIF1 and oxygen sensing in the brain. Nat Rev Neurosci. 2004;5:437-448.

8. Valable S, Petit E, Roussel S, et al. Complementary information from magnetic resonance imaging and ${ }^{18} \mathrm{~F}$-fluoromisonidazole positron emission tomography in the assessment of the response to an antiangiogenic treatment in a rat brain tumor model. Nucl Med Biol. 2011;38:781-793.

9. Carmeliet P, Jain RK. Angiogenesis in cancer and other diseases. Nature. 2000;407:249-257.

10. Aum DJ, Kim DH, Beaumont TL, Leuthardt EC, Dunn GP, Kim AH. Molecular and cellular heterogeneity: the hallmark of glioblastoma. Neurosurg Focus. 2014;37:E11.

11. Szeto MD, Chakraborty G, Hadley J, et al. Quantitative metrics of net proliferation and invasion link biological aggressiveness assessed by MRI with hypoxia assessed by FMISO-PET in newly diagnosed glioblastomas. Cancer Res. 2009; 69:4502-4509.

12. Swanson KR, Chakraborty G, Wang $\mathrm{CH}$, et al. Complementary but distinct roles for MRI and ${ }^{18} \mathrm{~F}$-fluoromisonidazole PET in the assessment of human glioblastomas. J Nucl Med. 2009;50:36-44.

13. Hirata K, Terasaka S, Shiga T, et al. ${ }^{18} \mathrm{~F}$-fluoromisonidazole positron emission tomography may differentiate glioblastoma multiforme from less malignant gliomas. Eur J Nucl Med Mol Imaging. 2012;39:760-770.

14. Le Bas J-F, Grand S, Kremer S, et al. Perfusion MR imaging for initial diagnosis and follow-up of brain tumors [in French]. Neurochirurgie. 2005;51:287-298.

15. Zagzag D, Zhong H, Scalzitti JM, Laughner E, Simons JW, Semenza GL. Expression of hypoxia-inducible factor $1 \alpha$ in brain tumors: association with angiogenesis, invasion, and progression. Cancer. 2000;88:2606-2618.

16. Emara M, Allalunis-Turner J. Effect of hypoxia on angiogenesis related factors in glioblastoma cells. Oncol Rep. 2014;31:1947-1953.

17. Gerstner ER, Zhang Z, Fink J, et al. ACRIN 6684: assessment of tumor hypoxia in newly diagnosed GBM using ${ }^{18}$ F-FMISO PET and MRI. Clin Cancer Res. 2016;22:5079-5086.

18. Chang CW, Chou TK, Liu RS, et al. A robotic synthesis of $\left[{ }^{18} \mathrm{~F}\right]$ fluoromisonidazole ([ ${ }^{18}$ F]FMISO). Appl Radiat Isot. 2007;65:682-686.

19. Spence AM, Muzi M, Swanson KR, et al. Regional hypoxia in glioblastoma multiforme quantified with $\left[{ }^{18} \mathrm{~F}\right]$ fluoromisonidazole positron emission tomography before radiotherapy: Correlation with time to progression and survival. Clin Cancer Res. 2008;14:2623-2630.

20. Collet S, Valable S, Constans JM, et al. $\left[{ }^{18} \mathrm{~F}\right]$-fluoro-l-thymidine PET and advanced MRI for preoperative grading of gliomas. Neuroimage Clin. 2015;8:448454.

21. Brasch R, Pham C, Shames D, et al. Assessing tumor angiogenesis using macromolecular MR imaging contrast media. J Magn Reson Imaging. 1997;7:68-74.

22. Lemasson B, Chenevert TL, Lawrence TS, et al. Impact of perfusion map analysis on early survival prediction accuracy in glioma patients. Transl Oncol. 2013;6:766-774.

23. Bulnes S, Bengoetxea H, Ortuzar N, et al. Angiogenic signalling pathways altered in gliomas: selection mechanisms for more aggressive neoplastic subpopulations with invasive phenotype. J Signal Transduct. 2012;2012:597915.

24. Huchet A, Fernandez P, Allard M, et al. Molecular imaging of tumor hypoxia [in French]. Cancer Radiother. 2009;13:747-757.

25. Bruehlmeier M, Roelcke U, Schubiger PA, Ametamey SM. Assessment of hypoxia and perfusion in human brain tumors using PET with ${ }^{18} \mathrm{~F}$-fluoromisonidazole and ${ }^{15} \mathrm{O}-\mathrm{H} 2 \mathrm{O}$. J Nucl Med. 2004;45:1851-1859.

26. Cher LM, Murone C, Lawrentschuk N, et al. Correlation of hypoxic cell fraction and angiogenesis with glucose metabolic rate in gliomas using ${ }^{18} \mathrm{~F}$-fluoromisonidazole, ${ }^{18}$ F-FDG PET, and immunohistochemical studies. J Nucl Med. 2006; 47:410-418.

27. Kawai N, Lin W, Cao W-D, et al. Correlation between ${ }^{18} \mathrm{~F}$-fluoromisonidazole PET and expression of HIF- $1 \alpha$ and VEGF in newly diagnosed and recurrent malignant gliomas. Eur J Nucl Med Mol Imaging. 2014;41:1870-1878. 